\title{
Effects of Systemic Administration of Iptakalim on Extracellular Neurotransmitter Levels in the Striatum of Unilateral 6-Hydroxydopamine-Lesioned Rats
}

\author{
Sen Wang', Li-fang Hu', Yun Zhang', Tao Sun', Ye-hong Sun', Su-yi Liu', Jian-hua Ding', Jie Wu',2 \\ and Gang Hu*, I \\ 'Department of Pharmacology and Neurobiology, Nanjing Medical University, Nanjing, Jiangsu Province, PR China; '2Division of Neurology, \\ Barrow Neurological Institute, St Joseph's Hospital and Medical Center, Phoenix, AZ, USA
}

\begin{abstract}
The function of ATP-sensitive potassium (KATP) channels in nigrostriatal pathway in Parkinson's disease (PD) was studied by employing a novel KATP channel opener iptakalim (Ipt). Apomorphine-induced rotation behavior test and microdialysis experiment were carried out in unilateral 6-hydroxydopamine (6-OHDA) lesioned rats. Behavior test showed that systemic administration of Ipt failed to significantly alleviate apomorphine-induced rotation in unilateral 6-OHDA-lesioned PD model rats. However, using in vivo microdialysis in this PD model rats, it was found that Ipt could increase extracellular dopamine levels in the lesioned side of the striatum and decrease dopamine levels in the intact side of the striatum. Meanwhile, Ipt had no influence on glutamate levels in the intact side, but it did decrease glutamate levels in the lesioned side of the striatum of PD rats. Additionally, in primary cultured rat astrocytes, 6-OHDA decreased overall glutamate uptake activity, but this decrease was recovered and glutamate uptake activity was restored by the opening of KATP channels induced by Ipt and pinacidil. The classical KATP channel blocker glibenclamide completely abolished the effects of Ipt and pinacidil. The present study suggests that (i) the function of KATP channels in the lesioned and intact nigrostriatal pathway is different in unilateral 6-OHDA-lesioned PD model rats. (ii) KATP channels regulate extracellular neurotransmitter levels in the striatum of unilateral 6-OHDAlesioned rats and may play neuroprotective roles due to their effects on glutamate transporters.

Neuropsychopharmacology (2006) 31, 933-940. doi: I 0. I038/sj.npp. I 300857; published online 3 August 2005
\end{abstract}

Keywords: Parkinson's disease; 6-hydroxydopamine; ATP-sensitive potassium channel; Iptakalim; neurotransmitter; glutamate uptake

\section{INTRODUCTION}

Parkinson's disease (PD), which is characterized by a selective loss of dopaminergic neurons in the substantia nigra pars compacta $(\mathrm{SNc})$, is one of the most prevalent neurodegenerative disorders. Loss of dopamine in the striatum triggers a cascade of functional changes that affect the basal ganglia (BG) network - where the balance of glutamate and dopamine is critical-ultimately contributing to severe motor symptoms such as tremor, rigidity, slowness of movement, and postural instability, all of which are observed in PD (Blandini et al, 2000).

ATP-sensitive potassium (KATP) channels, which link cell metabolism to its membrane potential, belong to a class of inwardly rectifying potassium channels that are activated

\footnotetext{
*Correspondence: Dr G Hu, Department of Pharmacology and Neurobiology, Nanjing Medical University, 140 Hanzhong Road, Nanjing 210029, Jiangsu, PR China, Tel: + 862586863169 , Fax: + 86 2586863 I08, E-mail: ghu@njmu.edu.cn

Received I February 2005 revised and accepted 29 June 2005

Online publication: I July 2005 at http://www.acnp.org/citations/ Npp070 105050072/default.pdf
}

by a decrease in the ATP/ADP ratio. KATP channels are widely distributed in the brain, with higher levels being localized in the BG (Mourre et al, 1990; Dunn-Meynell et al, 1998). Although KATP channels in many brain cell types are normally closed and do not contribute to resting membrane potentials, membrane hyperpolarization occurs when these channels are activated by selective openers or by decreased levels of oxygen or glucose. Previous studies have suggested that the activation of KATP channels inhibits neurotransmitter release, including glutamate (Lee et al, 1996), $\gamma$ aminobutyric acid (GABA) (Maneuf et al, 1996), and dopamine (Neusch et al, 2000; Zhu et al, 1999) in the BG. Studies have also indicated that KATP channels take part in regulation of the function of the substantia nigra (SN): dopamine was found to regulate a sulphonylurea-sensitive potassium channel (Greif et al, 1995; McGroarty and Greenfield, 1996) in dissociated cells from the SN, and the selective KATP channel blocker tolbutamide antagonized $\mathrm{D}_{2}$ receptor agonist-induced hyperpolarization (Roeper et al, 1990).

Previous studies have mostly focused on the physiological role of KATP channels in the striatum, SN, pallidum, and 
other BG nuclei, including neurotransmitter release; however, no previous investigation has focused on the role of KATP channels in BG function in pathological conditions, such as PD. Iptakalim hydrochloride (Ipt), a lipophilic para-amino compound with low molecular weight, has been demonstrated to be a novel KATP channel opener (KCO) through extensive pharmacological, electrophysiologi$\mathrm{cal}$, biochemical studies, and receptor binding tests (Wang 2003; Wang et al, 2004; Yang et al, 2004). Compared to other classical KCOs, Ipt easily crosses the bloodbrain barrier and there is no apparent difficulty in delivering the drug to the central nervous system. Therefore, the first aim of this study was to investigate the effects of systemic administration of Ipt on apomorphine-induced rotation behavior and the levels of extracellular dopamine and glutamate in striatum of unilateral 6-hydroxydopamine (6-OHDA)-lesioned rats by microdialysis experiment.

Glutamate, an excitatory neurotransmitter, is the most abundant neurotransmitter in the mammalian central nervous system, exerting several essential physiological functions (Matute et al, 2002). However, overstimulation of the glutamatergic system promotes excitotoxicity and is implicated in the pathology of PD (Hazell et al, 1997). Hence, modulation of extracellular glutamate levels determines its physiological and excitotoxic actions. Clearance of extracellular glutamate is mainly accomplished by glutamate transporters on astrocytes via a sodium-dependent mechanism (Anderson and Swanson, 2000). Astrocytic glutamate transporter plays a major role in regulating glutamatergic transmission and maintains extracellular glutamate concentrations below neurotoxic levels (Frizzo et al, 2001). A previous study performed in our lab suggested that 6-OHDA significantly decreases glutamate uptake without affecting primary astrocyte cell viability, which may underlie 6-OHDA-induced neurotoxicity (Zhang et al, 2004). A recent study has also indicated that the opening of KATP channels induced by Ipt affects multiple pathways of the glutamatergic system, limiting glutamate release and receptor actions, which are involved in excitotoxicity (Wang et al, 2004). The second aim of this study, therefore, was to investigate the effects of Ipt on 6OHDA-induced glutamate uptake decrease in primary astrocytes prepared from rats.

\section{MATERIALS AND METHODS}

\section{Animals and Reagents}

A total of 100 male Sprague-Dawley rats $(180-220 \mathrm{~g})$ were used in the present study and were housed with food and water available ad libitum. A $12 \mathrm{~h}$ light/dark cycle was used with lights being turned on at 0630. Postnatal (1-2-day-old) Sprague-Dawley rats were used to culture primary astrocytes. All experiments were carried out in strict accordance with the National Institutes of Health Guide for the Care and Use of Laboratory Animals. 6-OHDA was purchased from Sigma (USA). Ipt hydrochloride (99.9\%) was synthesized and provided by the Institute of Pharmacology and Toxicology, Academy of Military Medical Sciences of China. O-phthaldialdehyde (OPA) was obtained from Fluka (Switzerland). Dulbecco's modified Eagle's medium was obtained from Gibco (Rockville, MI, USA). D,L- $\left[{ }^{3} \mathrm{H}\right]$ glutamate was purchased from the Chinese Atomic Nucleus Research Institute (Beijing, China). All other reagents were provided by standard commercial institutes.

\section{Surgery and Drug Administration}

Rats were anesthetized using $2 \%$ sodium pentobarbital $(40 \mathrm{mg} / \mathrm{kg}$, i.p.) and placed in a stereotaxic apparatus (Stoelting Instruments, USA). Unilateral injection of 6OHDA $(8 \mu \mathrm{g} 6$-OHDA hydrochloride in $4 \mu \mathrm{l}$ of $0.02 \%$ ascorbic acid saline solution) was performed in the right SNc (coordinates from bregma: AP, $-5.3 \mathrm{~mm}$; $\mathrm{ML}$, $+1.8 \mathrm{~mm}$; DV, $-7.8 \mathrm{~mm}$; Paxinos and Watson, 1986) by means of a Hamilton syringe $(0.46 \mathrm{~mm}$ in diameter $)$ at a rate of $1 \mu \mathrm{l} / \mathrm{min}$, the needle was left in the place for $10 \mathrm{~min}$. Sham-lesioned rats were infused with $4 \mu \mathrm{l}$ saline containing $0.02 \%$ ascorbic acid into the right $\mathrm{SNc}$ and served as controls. At 3 weeks after the lesion, the animals' tendency to rotate in response to apomorphine $(0.5 \mathrm{mg} / \mathrm{kg}$, s.c. $)$ was tested. Contralateral rotations induced by apomorphine were measured two times at weekly intervals. Only animals showing at least 7 turns/min in both tests were included in this study. Successful model rats were divided into five groups: control model rats, an L-DOPA $10 \mathrm{mg} / \mathrm{kg}$ group, an Ipt $0.75 \mathrm{mg} / \mathrm{kg}$ group, an Ipt $1.5 \mathrm{mg} / \mathrm{kg}$ group, and an Ipt $3.0 \mathrm{mg} / \mathrm{kg}$ group. Later, L-3,4-dihydroxyphenylalanine (LDOPA, $10 \mathrm{mg} / \mathrm{kg} /$ day, p.o.) and Ipt $(0.75,1.5,3.0 \mathrm{mg} / \mathrm{kg} /$ day, p.o.) were administered, respectively, for 21 days. Shamoperated and control model rats received saline. At the end of each week, apomorphine $(0.5 \mathrm{mg} / \mathrm{kg}$, s.c.) was administered to test any change in the rotation behavioral of 6-OHDA-lesioned rats.

\section{Microdialysis Experiments}

At the end of the second week after drug administration and under pentobarbital anesthesia, rats were placed in a stereotaxic apparatus and implanted with a stainless steel dialysis guide cannula in both sides of the striatum (coordinates from bregma: AP, $+1.0 \mathrm{~mm}$; ML, $\pm 2.9 \mathrm{~mm}$; DV, $-3.5 \mathrm{~mm}$; Paxinos and Watson, 1986). The guide cannula was cemented in place using dental acrylic, which was applied to three stainless steel screws, which had previously been tapped into the skull of the rats. After the rats had recovered from surgery for 7 days, the concentric microdialysis probes (MD-2200, $2 \mathrm{~mm}$ membrane Bioanalytical system, Inc., Indiana, USA) were inserted through the guide cannula into the striatum. Artificial cerebrospinal fluid (ACSF; $\mathrm{NaCl} 140 \mathrm{mM}, \mathrm{CaCl}_{2} 1.4 \mathrm{mM}, \mathrm{MgCl}_{2} 1.2 \mathrm{mM}$, $\mathrm{KCl} 2.7 \mathrm{mM}$, and glucose $5 \mathrm{mM}$ ) was infused through the probe via a syringe pump (Bioanalytical systems, Inc., Indiana, USA), but before use the ACSF was adjusted to $\mathrm{pH}$ 7.4 using phosphate-buffered saline and was filtered by $0.22 \mu \mathrm{m}$ vacuum filtration. Perfusion with ACSF started $12 \mathrm{~h}$ after probe insertion and lasted for $1 \mathrm{~h}$ at a rate of $2 \mu \mathrm{l}$ per min for equilibrium. Afterward, samples were collected for $40 \mathrm{~min}$ and each collected sample was aliquoted into two microcentrifuge tubes with $40 \mu \mathrm{l}$ in each tube for analysis of dopamine and glutamate levels. All samples were frozen at $-70^{\circ} \mathrm{C}$ before they were analyzed. 


\section{Analysis of Dopamine and Glutamate}

Dialysate samples were assayed for dopamine by highperformance liquid chromatography (HPLC) with electrochemical detection (ECD). HPLC/ECD consisted of BAS LC-4C, a reversed-phase C18 column (Ultrasphere ODS $4.6 \times 250 \mathrm{~mm}^{2}, 5 \mu \mathrm{m}$, Japan), chromograph interface DA-5, and solvent delivery system. The mobile phase consisted of citrate $0.1 \mathrm{M}, \mathrm{Na}_{2} \mathrm{HPO}_{4} 0.075 \mathrm{M}$, EDTA $0.1 \mathrm{M}$, 1-heptanesulfonic acid $1.0 \mathrm{mM}$, and methanol $10 \%, \mathrm{pH} 3.9$. The concentration of glutamate was determined by HPLC with fluorescent detection. The HPLC-fluorescent detector system (Shimadzu Corp, Tokyo, Japan) consisted of Shimadzu HPLC, a reverse phase C18 column (Ultrasphere ODS $4.6 \times 250 \mathrm{~mm}^{2}, 5 \mu \mathrm{m}$, Japan), fluorescence HPLC monitor RF-530, and a liquid chromatograph LC-6A. The mobile phase consisted of $0.1 \mathrm{M} \mathrm{Na} \mathrm{HPO}_{4} \cdot 12 \mathrm{H}_{2} \mathrm{O}, 0.1 \mathrm{mM}$ edetic acid, methanol 30\%, pH 6.04. The emission and excitation wavelengths were set at 425 and $338 \mathrm{~nm}$, respectively. The precolumn derivation solution contained OPA $(20 \mathrm{mM}), \beta$ mercaptoethanol (2 mM), tetraborate $(25 \mathrm{mM}), 50 \%$ methanol, $\mathrm{pH}$ 9.6. Samples were mixed with an equivalent volume of derivation solution and were incubated at room temperature $\left(21 \pm 1^{\circ} \mathrm{C}\right)$ for $4 \mathrm{~min}$. The derived reaction solutions were used to assay glutamate concentrations at $37^{\circ} \mathrm{C}$ with a flow rate of $0.8 \mathrm{ml} / \mathrm{min}$. At the conclusion of experiments, rats were given an overdose of pentobarbital ( $>50 \mathrm{mg} / \mathrm{kg}$, i.p) and perfused intracardially with $10 \%$ formalin. Coronal sections ( $40 \mu \mathrm{m}$ thick) of the brain were cut to verify the location of the probe. Samples obtained from rats in which the probe was not correctly positioned were excluded from analysis.

\section{Primary Culture of Astrocytes and Assay of Astrocytic Glutamate Uptake Activity}

Rat primary astrocytes were prepared from whole brains of postnatal (1-2-day-old) Sprague-Dawley rats and were plated on poly-D-lysine precoated cell culture flasks containing Dulbecco's modified Eagle's medium (10\% fetal calf serum, $100 \mathrm{U} / \mathrm{ml}$ penicillin, and $100 \mu \mathrm{g} / \mathrm{ml}$ streptomycin). The cells were grown in a humidified atmosphere of $5 \% \quad \mathrm{CO}_{2} / 95 \%$ air at $37^{\circ} \mathrm{C}$. When the astrocytes reached confluence, the cells were passaged by trypsinization and plated at a density of $10^{6}$ cells/well on 24 -well culture plates in a final volume of $1 \mathrm{ml}$ Dulbecco's modified Eagle's medium, and were grown in a humidified atmosphere of $5 \%$ $\mathrm{CO}_{2} / 95 \%$ air at $37^{\circ} \mathrm{C}$. At 2 days later, the astrocytes were used for experimentation. Immunocytochemical analyses have shown that this method produces cultures comprising $>95 \%$ glial fibrillary acid protein-positive astrocytes.

Glutamate uptake measurements were performed as previously described by Pines and Kanner (1990) with little modification. Briefly, after exposure of cultures to 6-OHDA for $24 \mathrm{~h}$, the culture medium was removed and HEPES buffered saline containing $\left[{ }^{3} \mathrm{H}\right]$ glutamate was added to each well (final concentration was $50 \mu \mathrm{M}$ ). Exactly 15 min later, glutamate uptake was terminated and cells were rapidly washed three times with ice-cold $\mathrm{NaCl}(0.9 \%)$. The cells were harvested and then solubilized in $0.2 \mathrm{ml} \mathrm{NaOH}(0.3 \mathrm{M})$ followed by immediate centrifugation $(5000 \mathrm{~g}$ for $5 \mathrm{~min}$ at $4{ }^{\circ} \mathrm{C}$ ). Radioactive and protein contents of the supernatant were determined by the liquid scintillation counting and Bradford methods, respectively.

\section{Statistics}

All data are presented as mean \pm SEM. For behavioral tests and glutamate uptake activity, a separate one-way analysis of variance (ANOVA) was carried out to determine the significance between groups by means of STATA 7.0, followed by the Scheffe test. For DA and glutamate analysis in microdialysis experiment, a nonparametric KruskalWallis test was used to analyze the significance between groups. When Kruskal-Wallis test showed a significant difference, further analysis was performed by Nemenyi test. $P$-values less than 0.05 were considered significant in all the tests.

\section{RESULTS}

\section{Behavioral Test}

Apomorphine-induced rotations in the model group were consistent throughout all of the experiments, suggesting the stabilization of rotation behavior of the unilateral 6-OHDAinduced $\mathrm{PD}$ model rats $\left(\mathrm{F}_{(3,33)}=0.01, P=0.9994\right)$. The results (Figure 1) showed that treatment of $\mathrm{PD}$ rats with L-DOPA $(10 \mathrm{mg} / \mathrm{kg})$ significantly alleviated apomorphineinduced rotations in rats from all 3 weeks compared to pretreatment $\left(\mathrm{F}_{(3,28)}=4.74, P=0.0085\right)$, but systemic administration of Ipt failed to reduce the apomorphineinduced rotation behavior significantly in the 3 weeks.

\section{Extracellular Dopamine and Glutamate Levels}

Figure 2a shows the effects of systemic administration of Ipt on extracellular dopamine levels in the striatum of 6OHDA-treated model rats. In the lesioned and intact side of the striatum of sham-operated rats, extracellular dopamine and glutamate concentrations were measured. These two neurotransmitter levels in each side were comparable, suggesting that the injection process did not affect SNc function. In unilateral 6-OHDA-lesioned rats, extracellular dopamine levels in the intact side did not change compared

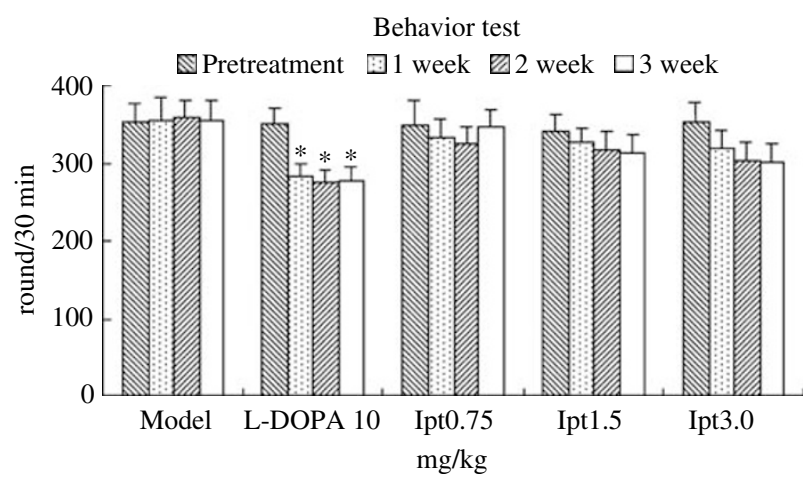

Figure I Effects of systemic administration of Ipt on rotation behavior induced by apomorphine in 6-OHDA-treated model rats. $n=10$ for model group and L-DOPA group, $n=12$ for other groups. Mean \pm SEM, $* P<0.05$ vs pretreatment of the same group. 
to sham-operated rats, while extracellular dopamine levels in the lesioned side of the striatum decreased significantly due to the injection of 6-OHDA into SNc $\left(\chi_{0.05,1}^{2}=5.333\right.$, $P=0.021)$. Administration of L-DOPA did not affect dopamine levels in either the lesioned or intact sides of the striatum, while systemic administration of Ipt (1.5 and $3.0 \mathrm{mg} / \mathrm{kg}$ ) decreased dopamine levels in the intact side of the striatum compared to the intact side of 6-OHDAlesioned rats $\left(\chi_{0.05,4}^{2}=14.905, P<0.0001\right)$. However, dopamine levels in the lesioned side of the striatum increased significantly compared to the lesioned side of 6-OHDAlesioned rats $\left(\chi_{0.05,4}^{2}=13.447, P<0.0001\right)$. Figure $2 \mathrm{~b}$ shows the effects of systemic administration of Ipt on extracellular glutamate levels in the striatum of 6-OHDA-treated model rats. In unilateral 6-OHDA-lesioned rats, a significant increase in extracellular glutamate levels in the lesioned side of the striatum was observed compared to the intact side $\left(\chi_{0.05,1}^{2}=5.333, P=0.021\right)$. Administration of $\mathrm{L}$-DOPA or Ipt $(0.75,1.5$ and $3.0 \mathrm{mg} / \mathrm{kg})$ did not affect glutamate levels in the intact side of the striatum. However, L-DOPA significantly decreased glutamate levels in the lesioned side of the striatum of PD rats, while systemic administration of Ipt $(0.75,1.5$, and $3.0 \mathrm{mg} / \mathrm{kg})$ abolished the increase of extracellular glutamate levels in the lesioned side of the striatum of parkinsonian rats $\left(\chi_{0.05,4}^{2}=15.503, P<0.0001\right)$.

\section{Effects of Ipt on 6-OHDA-Induced Decrease of Astrocytic Glutamate Uptake Activity}

Considering that astrocytes play important role in regulating extracellular glutamate levels, experiments were carried
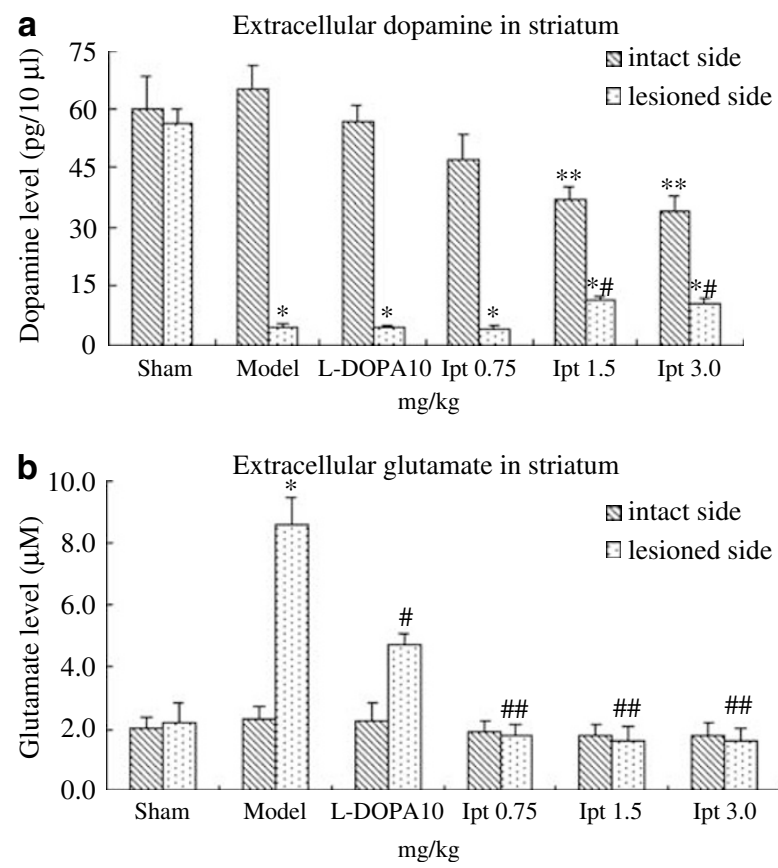

Figure 2 Effects of systemic administration of lpt on extracellular dopamine (a) and glutamate (b) in the striatum of 6-OHDA-treated model rats. $n=4$ for sham and model groups, $n=5$ for the other groups, mean \pm SEM, $* P<0.0$ I vs the intact side of the same group, ${ }^{*} * P<0.05$ vs the intact side of the model group, ${ }^{\#} P<0.05$ vs the lesioned side of the model group, ${ }^{\# \#} P<0.0$ I vs the lesioned side of the model group. out to explore the mechanisms underlying the effects of Ipt decreasing extracellular glutamate levels in lesioned side of striatum in 6-OHDA model rats on astrocytes. Isolated astrocytes were incubated in vitro with Ipt or pinacidil without or in the presence of 6-OHDA $(50 \mu \mathrm{M})$ for $24 \mathrm{~h}$. Astrocytic glutamate uptake activity was determined by measuring intracellular concentrations of $\left[{ }^{3} \mathrm{H}\right]_{\mathrm{D}, \mathrm{L}-\text { glutamate }}$ using isotope techniques. Table 1 shows the effects of Ipt on normal and impaired astrocytic glutamate uptake activity $\left(\mathrm{F}_{(11,36)}=9.842, P<0.001\right)$. Ipt alone did not affect astrocytic glutamate uptake activity at lower concentrations $(1,10,100 \mathrm{nM})$, but it increased glutamate uptake activity at higher concentrations $(1,10 \mu \mathrm{M})$. After preincubation of cultured astrocytes with different concentrations of Ipt for $20 \mathrm{~min}$ following incubation with 6-OHDA $(50 \mu \mathrm{M})$ for $24 \mathrm{~h}$, glutamate uptake was assessed. 6-OHDA decreased glutamate uptake in primary cultured astrocytes, and the inhibition rate was $20 \%$. Ipt $(10,100 \mathrm{nM} ; 1,10 \mu \mathrm{M})$ countered the effects of 6-OHDA on glutamate uptake by increasing uptake from $41.4 \pm 1.5$ to $51.6 \pm 2.1,52.9 \pm 1.5$, $61 \pm 8$, and $53 \pm 4$, respectively.

\section{Effects of Ipt or Pinacidil on 6-OHDA-Induced Decrease of Astrocytic Glutamate Uptake Activity and the Antagonistic Effects of Glibenclamide}

Isolated astrocytes were incubated in vitro with Ipt or pinacidil and 6-OHDA $(50 \mu \mathrm{M})$ without or in the presence of glibenclamide $(20 \mu \mathrm{M})$ for $24 \mathrm{~h}$. Astrocytic glutamate uptake activity was determined by measuring intracellular concentrations of $\left[{ }^{3} \mathrm{H}\right]_{\mathrm{D}, \mathrm{L} \text {-glutamate }}$ using isotope techniques. Table 2 shows the reversed effects of Ipt or pinacidil (Pina) on astrocytic glutamate uptake activity and the antagonistic effects of glibenclamide (Gli) $\left(\mathrm{F}_{(7,40)}=7.649, P<0.001\right)$. 6OHDA inhibited glutamate uptake in primary cultured

Table I Effects of Ipt on Normal and Impaired Astrocytic Glutamate Uptake Activity

\begin{tabular}{|c|c|}
\hline Group & {$\left[{ }^{3} \mathrm{H}\right] \mathrm{D}, \mathrm{L}-$ Glutamate/nmol/min/mg (pro) } \\
\hline Control & $51 \pm 4$ \\
\hline Ipt I nM & $48.1 \pm 1.2$ \\
\hline Ipt $10 \mathrm{nM}$ & $48.2 \pm 2.2$ \\
\hline Ipt $100 \mathrm{nM}$ & $48.0 \pm 1.4$ \\
\hline lpt $\mid \mu M$ & $60 \pm 4 *$ \\
\hline Ipt $10 \mu \mathrm{M}$ & $58 \pm 5^{*}$ \\
\hline 6-OHDA & $41.4 \pm 1.5^{*}$ \\
\hline Ipt I nM+6-OHDA & $48.3 \pm 2.6$ \\
\hline Ipt I0 nM+6-OHDA & $51.6 \pm 2.1^{\#}$ \\
\hline Ipt $100 \mathrm{nM}+6-\mathrm{OHDA}$ & $52.9 \pm 1.5^{\# \#}$ \\
\hline Ipt $\mid \mu M+6-O H D A$ & $61 \pm 8^{\# \#}$ \\
\hline Ipt $10 \mu \mathrm{M}+6-\mathrm{OHDA}$ & $53 \pm 4^{\# \#}$ \\
\hline
\end{tabular}

Isolated astrocytes from the cerebral cortexes of I- to 2-day-old SpragueDawley rats were incubated with lpt of various concentrations in vitro without or in the presence of 6-OHDA $(50 \mu \mathrm{M})$ for $24 \mathrm{~h}$. Astrocytic glutamate uptake activity was determined by measuring intracellular concentration of ${ }^{3} \mathrm{H}$-labeled $D, L$-glutamate using isotope techniques. $n=4$ for each group, mean $\pm S E M$, ${ }^{*} P<0.05$ vs the control group; ${ }^{\#} P<0.05,{ }^{\#} P<0.01$ vs the 6-OHDA group. 
Table 2 Reversed Effects of lpt or Pinacidil (Pina) on Astrocytic Glutamate Uptake Activity and the Antagonistic Effects of Glibenclamide (Gli)

\begin{tabular}{|c|c|}
\hline Group $/ \mu \mathrm{M}$ & {$\left[{ }^{3} \mathrm{H}\right] \mathrm{D}, \mathrm{L}-$ Glutamate/nmol/min/mg (pro) } \\
\hline Control & $71 \pm 4$ \\
\hline 6-OHDA & $61 \pm 9^{@}$ \\
\hline $6-O H D A+$ lpt I & $79 \pm 6^{\# \#}$ \\
\hline 6-OHDA+lpt 10 & $78 \pm 5^{\# \#}$ \\
\hline 6-OHDA+Pina 10 & $75 \pm 4^{\#}$ \\
\hline Gli+lpt I+6-OHDA & $69 \pm 4 * \$$ \\
\hline Gli+lpt 10+6-OHDA & $63 \pm 5^{1, \$}$ \\
\hline Gli+Pina 10+6-OHDA & $67 \pm 5^{8, \$}$ \\
\hline
\end{tabular}

Isolated astrocytes were incubated with lpt or Pina and 6-OHDA $(50 \mu \mathrm{M})$ with no or in the presence of glibenclamide $(20 \mu \mathrm{M})$ in vitro for $24 \mathrm{~h}$. Astrocytic glutamate uptake activity was determined by intracellular concentration of $\left[{ }^{3} \mathrm{H}\right] \mathrm{D}, \mathrm{L}$-glutamate, using isotope techniques. $n=6$ for each group, mean $\pm \mathrm{SEM}$, ${ }^{*} P<0.0$ I vs lpt $(\mid \mu \mathrm{M})+6-\mathrm{OHDA}$ group; $: P<0.05$ vs lpt $(10 \mu \mathrm{M})+6-\mathrm{OHDA}$ group; ${ }^{\circledR} P<0.05$ vs Pina $(10 \mu \mathrm{M})+6-\mathrm{OHDA}$ group; ${ }^{\$} P>0.05,{ }^{\#} P<0.05$,

${ }_{\# \#} P<0.0$ I vs 6-OHDA group; ${ }^{@} P<0.05$ vs control group.

astrocytes, and the inhibition rate was $15 \%$. Ipt $(1,10 \mu \mathrm{M})$ $(P<0.01)$ or pinacidil $(10 \mu \mathrm{M})$ reversed the effects of 6 OHDA by enhancing astrocytic glutamate uptake activity by about $10 \%$. The selective KATP channel blocker glibenclamide $(20 \mu \mathrm{M})$ completely abolished the effects of Ipt and pinacidil.

\section{DISCUSSION}

The present study demonstrates for the first time the effects of systemic administration of Ipt, a novel KCO, on the rotational behavior and extracellular neurotransmitter levels in the striatum of unilateral 6-OHDA-lesioned rats. Systemic administration of Ipt exerted different regulatory effects on extracellular dopamine and glutamate levels between the lesioned and intact side of striatum. These results indicated that the functional characteristics of KATP channels between the lesioned and intact nigrostriatal pathway are different in unilateral 6-OHDA-lesioned PD model rats. Moreover, 6-OHDA decreased glutamate uptake in primary astrocytes while Ipt effectively reversed the 6OHDA-induced decrease, which was in turn abolished by the specific KATP channel blocker glibenclamide. These results provide novel evidence for a role of KATP channels in the lesioned nigralstriatal pathway of PD model rats and suggest that KCOs may serve as new neuroprotective agents for the treatment of PD.

A number of animal models have been developed to selectively destroy the mesostriatal dopamine system, and they are thought to mimic at least some aspects of PD (Betarbet et al, 2002). One such model utilizes unilateral injection of the neurotoxin 6-OHDA into the $\mathrm{SNc}$ to selectively destroy mesostriatal dopamine inputs (Tolwani et al, 1999) and has been proven useful for pharmacological screening of agents that have effects on dopamine and its receptors. Degeneration of dopaminergic neurons in the $\mathrm{SNc}$ results in decreased dopaminergic activity in the striatum while increasing glutamatergic activity in the subthalamic nucleus (STN). This overactivity can lead to increased glutamate-mediated excitation in BG output regions, such as the substantia nigra pars reticulata $(\mathrm{SNr})$ and globus pallidus internus (GPi) or the rodent homologue, the entopeduncular nucleus, which may in turn lead to reduced thalamocortical feedback and the subsequent appearance of akinesia (Blandini et al, 2000). Since neurochemical deficits and the functional consequences of dopaminergic cell loss on local BG circuitry are well characterized, strategies can be designed to either improve cell survival or modify functional activity in the damaged BG circuitry. Therefore, targeting the regulation of neurotransmission, including dopamine and glutamate, may be one approach in the symptomatic treatment of PD.

Although experiments such as injecting tolbutamide (a sulfonylurea which block KATP channels) into the SN or glibizide (a sulfonylurea) into the dosal pallidum have indicated that modulation of BG KATP channels produces behavioral responses (Levesque and Greenfield, 1991; Maneuf et al, 1996), it is unclear whether modulation of KATP channels by systemic administration of a KCO can result in behavioral changes in the classical 6-OHDA PD rat model. After unilateral injection of 6-OHDA into the SNc, successful parkinsonian rats were systemically administrated Ipt or L-DOPA for three consecutive weeks. Administration of small dose of L-DOPA $(10 \mathrm{mg} / \mathrm{kg})$ supplying the precursor of dopamine, which may increase the dopamine concentration in striatum and balance the imbalance of sensitivity of dopamine receptor between intact side and lesioned side, alleviating apormphineinduced rotation behavior (Cao et al, 2003; Murer et al, 1998; Nutt and Hloford, 1996). Systemic administration of L-DOPA in the present study significantly alleviated apomorphine-induced rotations during all 3 weeks. However, unilateral denervation in striatum followed 6-OHDA lesion was so complex that only supplying L-DOPA could not completely reverse apomorphine-induced rotation, which may be the reason for those PD model rats receiving chronic L-DOPA still exhibited $>7$ rounds/min rotation behavior. Unilateral 6-OHDA injection represents the socalled 'hemiparkinson model' (Perese et al, 1989), which is characterized by an asymmetric motorcircling behavior after administration of dopaminergic drugs, due to the pathological imbalance between the lesioned and unlesioned striatum (Betarbet et al, 2002).

In our previous studies, we have found that Ipt significantly alleviated haloperidol-induced catalepsy and hypolocomotion (Wang et al, 2005), and improved parkinsonian symptoms in rotenone-prepared PD model rats (Yang et al, 2005). These results clearly indicated that Ipt exhibited significant neuroprotection in PD model rats. In the present study, Ipt only revealed a weaker effect in animal behavior rather than alleviate apomorphine-induced rotation significantly, due to severe injury of dopaminergic neurons in this kind of PD model rats. It was reported that there was about $90 \%$ doparminergic neurons loss in SNc in the PD model rats used in our study (Carman et al, 1991; Ronald et al, 2002). Obviously, Ipt played a potent role on neurotransmission, which was certainly beneficial to alleviating PD. The above-mentioned results suggested Ipt probably acted on BG glutamate-dopamine system to 
rebalance the dopamine imbalance between lesioned and intact striatum, and could be of important clinical relevance to treatment of PD. Therefore, to further investigate the effects of systemic administration of Ipt on extracellular neurotransmitter levels, such as dopamine and glutamate, in the striatum, a microdialysis probe was implanted into the terminal fields of the nigrostriatal projection.

As previously mentioned, KATP channels actively take part in regulation of neurotransmitter release in the BG, and the physiological role of KATP channels in the nigrostriatal pathway has been extensively studied (Lee et al, 1996; Maneuf et al, 1996; Neusch et al, 2000), yet the role they play in the dysfunctional pathway remains unclear. In conscious, freely moving 6-OHDA-lesioned rats chronically administered Ipt (1.5 and $3.0 \mathrm{mg} / \mathrm{kg}$ ), extracellular dopamine levels in the intact side of the striatum were significantly lower when compared to parkinsonian rats that only received saline, which was in accordance with previous studies that demonstrated that cromakalim (a KCO) can decrease extracellular dopamine levels in the striatum of normal rats (Zhu et al, 1999). Avshalumov and Rice (2004) also pointed out that opening of KATP channels either by endogenous $\mathrm{H}_{2} \mathrm{O}_{2}$ or KCOs will result in decreased dopamine release. KATP channels are found to express in presynaptically in dopaminergic neuron terminal projected from SNc, and mediate neurotransmitter release. By opening KATP channels, KCOs induce hyperpolarization of neuron terminals presynaptically, thereby affecting neurotransmitter release, and this may play an important role in the observed Ipt-induced decrease of dopamine levels in the intact side of the striatum.

Administrated with L-DOPA $10 \mathrm{mg} / \mathrm{kg} /$ day for 3 weeks, those PD model rats exhibited decreased rotational behavior induced by apomorphine. Resulting from the temporal effects of L-DOPA, we failed to detect the increase of extracellular dopamine levels in the striatum. On the other hand, extracellular dopamine levels in the lesioned side of the striatum of parkinsonian rats that received systemic administration of Ipt were significantly higher when compared to the lesioned side of the striatum of control PD model rats that only received saline, which was beneficial to the impaired function of BG. These results also indicate that Ipt play a different role in regulating extracellular dopamine concentrations in the intact and lesioned nigrostriatal pathway. Avshalumov et al pointed out that striatum extracellular dopamine concentrations were mediated by multiple factors, among which glutamate exerted important effects: acting on AMPA receptors located in nondopaminergic neuron terminal, glutamate inhibited dopamine release from dopaminergic terminals, by generating $\mathrm{H}_{2} \mathrm{O}_{2}$ to regulating KATP channels in dopaminergic terminals (Avshalumov et al, 2003; Avshalumov and Rice, 2004). Ipt decreased extracellular glutamate levels that increased after 6-OHDA lesion in striatum; therefore, the effects of Ipt increasing extracellular dopamine levels probably result in its effects on decreasing extracellular glutamate concentrations. Our previous study revealed that in lesioned side of PFC, hippocampus, and striatum in unilateral 6-OHDA-lesioned rats, expression of certain KATP channel subunits increased significantly when compared to that of intact side, also indicating that the function of KATP channels in the lesioned and intact nigrostriatal pathway is different in unilateral 6-OHDAlesioned PD model rats (Wang et al, 2005), and this may be another reason for different role of Ipt mentioned above. However, the role of Ipt and other KCOs in regulating neurotransmitter levels in other BG nuclei, such as the GPi, STN, and thalamus in pathological conditions seems to be interesting and remains unclear.

In the lesioned side of the striatum of 6-OHDA-leisoned rats, not only did extracellular dopamine levels decrease but also extracellular glutamate levels significantly increased when compared to the intact side of the striatum, which was consistent with previous studies (Calabresi et al, 1993; Meshul et al, 1999; Jonkers et al, 2002). Increased extracellular glutamate in the striatum may contribute to both excitotoxicity of dopaminergic neuron terminals from the SNc and dysfunction of the BG (Hazell et al, 1997). Extracellular glutamate levels in the lesioned side of the striatum of 6-OHDA-lesioned rats that received systemic administration of Ipt were significantly lower while glutamate levels in the intact side of the striatum did not significantly change when compared to control model rats that only received saline. The effects of Ipt in decreasing extracellular glutamate levels that were originally higher in the lesioned side of the striatum of 6-OHDA-lesioned rats are obviously very interesting and exciting due to the fact that increased glutamate may be harmful to neurons (Blandini et al, 2000); hence, Ipt may potentially play a neuroprotective role in neurological diseases. Elucidating the precise mechanism(s) of Ipt's induced decrease in extracellular glutamate levels in the lesioned side of the striatum of unilateral 6-OHDA-lesioned rats is of great importance, especially considering the potential of KCOs at these receptors in the treatment of neurodegeneration.

Two main processes are involved in maintaining physiological glutamate concentrations at the synaptic cleft: one regulates glutamate release from the presynaptic membrane and the other regulates glutamate uptake, which is mainly mediated by astrocytes located near the synapse. As glutamate transporter functional deficiency is characteristic of many neurodegenerative diseases (Vandenberg, 1998; Ferrarese et al, 2000), including PD, researchers have been looking for drugs that effectively stimulate glutamate transporter. Previous studies suggested that functional KATP channels exists in both astrocytes in adult rats and primary astrocytes (Brockhaus and Deitmer, 2000; Zhou et al, 2002). Therefore, in order to further explore the mechanisms underlying the effects of Ipt on extracellular glutamate levels in the striatum of unilateral 6-OHDAlesioned rats, the effects of Ipt on glutamate uptake activity in primary astrocytes were studied. 6-OHDA decreased glutamate uptake activity in primary astrocytes, which was consistent with previous studies that glutamate uptake by striatum synaptosomes prepared from the lesioned side of unilateral 6-OHDA-lesioned rats decreased significantly when compared to the intact side (Meng et al, 2003) and explains in part the mechanisms underlying elevated extracellular glutamate levels found in the lesioned side of the striatum of unilateral 6-OHDA-lesioned rats.

Ipt reversed the 6-OHDA-induced decrease of glutamate uptake activity while another classical KCO, pinacidil, had the same effects. The effects of Ipt and pinacidil were abolished by the KATP channel blocker, glibenclamide, 
which also supports the results obtained from microdialysis, and indicates that Ipt abolished the increase of extracellular glutamate levels in the lesioned side of the striatum of parkinsonian rats via glutamate transporters. Activity of $\mathrm{Na}^{+}$-dependent glutamate transporters is governed by both thermodynamic and kinetic factors, such as $\mathrm{Na}^{+} / \mathrm{K}^{+}$ gradients, binding affinities (Anderson and Swanson, 2000). Since the driving forces are all supplied by ATP hydrolysis, severe ATP depletion, such as resulting from 6-OHDAinduced CX I inhibition will lead to cessation of glutamate uptake, even reversal of glutamate transporters, which further increases the extracellular accumulation of glutamate (Madl and Burgesser, 1993). Opening of $\mathrm{K}_{\mathrm{ATP}}$ channels may result in the preservation of intracellular ATP production, hence, subsequently elevate activity of glutamate transporter. Moreover, activation of $\mathrm{K}_{\mathrm{ATP}}$ channels leads to almost immediate activation of protein kinase $\mathrm{C}$ (Kis et al, 2003). Activator of protein kinase C such as phorbol esters rapidly stimulates glutamate transporter activity (Davis et al, 1998; Dowd and Robinson, 1996; Yao et al, 2005). However, it is interesting that Ipt alone can increase glutamate uptake activity in astrocytes without influencing extracellular glutamate levels in the striatum of the intact side of 6-OHDA-lesioned rats, which may be due to the different in vivo and in vitro experimental protocols. That glutamate uptake can be enhanced via KATP channel opening suggests new directions for the development of novel drugs. In the initial years of treatment, L-DOPA is highly successful in reversing the parkinsonian symptoms. However, following several years of treatment many complications such as abnormal involuntary movements are observed. Compared to L-DOPA, Ipt not only increased extracellular dopamine concentration but also decreased glutamate level in lesioned side of striatum in 6-OHDA unilateral lesioned rats. Increased dopamine in lesioned side of striatum leads to behavior improvement, while decreased glutamate level may result in neuroprotection. And so, we hypothesized that Ipt may be a promising compound for treating PD.

In conclusion, Ipt has different effects on neurotransmitter levels in both the lesioned and intact sides of the striatum of 6-OHDA-lesioned rats, which would imply that the function of KATP channels in the lesioned and intact nigrostriatal pathway is different in unilateral 6-OHDAlesioned PD model rats. As indicated by the present study, it is reasonable to conclude that KCOs, at least the novel KATP channel opener Ipt, can regulate dopamine and glutamate levels in both the lesioned and intact sides of the striatum of 6-OHDA-lesioned rats. These results give rise to the exciting possibility that KATP channels in the striatum and selective KCOs that can freely cross the blood-brain barrier could provide an entirely new approach to the treatment of PD.

\section{ACKNOWLEDGEMENTS}

The research was supported in part by grants from the National Natural Science Foundation of China (No. 39970846), the Key Project of Jiangsu Health Department (No. K200501), the Key Project of Natural Science Foundation of Jiangsu Educational Council and Specialized
Research Fund for the Doctoral Program of Higher Education (No. 20040312004). Dr Kevin Ellsworth, in Barrow Neurological Institute (Phoenix AZ, USA), helped to correct English writing.

\section{REFERENCES}

Anderson CM, Swanson RA (2000). Astrocyte glutamate transporter: review of properties, regulation, and physiological functions. Glia 32: 1-14.

Avshalumov MV, Chen BT, Marshall SP, Penã DM, Rice ME (2003). Glutamate-dependent inhibition of dopamine release in striatum is mediated by a new diffusible messenger, $\mathrm{H}_{2} \mathrm{O}_{2}$. J Neurosci 23: 2744-2750.

Avshalumov MV, Rice ME (2004). Activation of ATP-sensitive $\mathrm{K}_{-}$(KATP) channels by $\mathrm{H}_{2} \mathrm{O}_{2}$ underlies glutamate-dependent inhibition of striatal dopamine release. PNAS 100: 11729-11734.

Betarbet RB, Sherer JT, Greenamyre J (2002). Animal models of Parkinson's disease. Bioessays 24: 308-318.

Blandini GF, Nappi C, Tassorelli E, Martignoni E (2000). Functional changes of the basal ganglia circuitry in Parkinson's disease. Prog Neurobiol 62: 63-88.

Brockhaus J, Deitmer JW (2000). Developmental downregulation of ATP-sensitive potassium conductance in astrocytes in situ. Glia 32: 205-213.

Calabresi P, Mercuri NB, Sancesario G, Bernardi G (1993). Electrophysiology of dopamine-denervated striatal neurons: Implications for Parkinson's disease. Brain 116: 433-452.

Cao F, Sun SG, Tong ET, Luo F (2003). Experimental study on the behavioral effects on rats with Parkinson's disease by different dose of Levodopa. J Apoplexy Nerv Dis 20: 14-16.

Carman LS, Gage FH, Shults CW (1991). Partial lesion of the substantia nigra: relation between extent of lesion and rotational behavior. Brain Res 553: 275-283.

Davis KE, Straff DJ, Weinstein EA, Bannerman PG, Correale DM, Rothstein JD et al (1998). Multiple signaling pathways regulate cell surface expression and activity of the excitatory amino acid carrier 1 subtype of Glu transporter in C6 glioma. J Neurosci 18: $2475-2485$.

Dowd LA, Robinson MB (1996). Rapid stimulation of EAAC1mediated Nacl-dependent L-glutamate transporter activity in C6 glioma by phorbol ester. J Neurochem 67: 508-516.

Dunn-Meynell AA, Rawson NE, Levin BE (1998). Distribution and phenotype of neurons containing the ATP sensitive potassium channel in rat brain. Brain Res 814: 41-54.

Ferrarese C, Begni B, Canevari C, Zoia C, Piolti R, Frigo M et al (2000). Glutamate uptake is decreased in platelets from Alzheimer's disease patients. Ann Neurol 47: 641-643.

Frizzo ME, Lara DR, Dahm KC, Prokopiuk AS, Swanson RA, Souza DO (2001). Activation of glutamate uptake by guanosine in primary astrocyte cultures. Neuroreport 12: 879-881.

Greif GJ, Lin YJ, Liu JC, Freedman JE (1995). Dopamine-modulated potassium channels on rat striatal neurons: specific activation and cellular expression. J Neurosci 15: 4533-4544.

Hazell AS, Itzhak Y, Liu H, Norenberg MD (1997). 1-Methyl-4phenyl-1,2,3,6-tetrahydropurikine (MPTP) decreases glutamate uptake in cultured astrocytes. J Neurochem 68: 2216-2219.

Jonkers N, Sarre S, Ebinger G, Michotte Y (2002). MK801 suppresses the L-DOPA-induced increase of glutamate in striatum of hemi-Parkinson rats. Brain Res 926: 149-155.

Kis B, Rajapakse NC, Snipes JA, Nagy K, Horiguchi T, Busija DW (2003). Diazoxide induces delayed pre-conditioning in cultured rat cortical neurons. J Neurochem 87: 969-980.

Lee K, Dixon AK, Rowe IC, Ashford ML, Richardson PJ (1996). The high-affinity sulphonylurea receptor regulates KATP channels in nerve terminals of the rat motor cortex. J Neurochem 66: 2562-2571. 
Levesque D, Greenfield SA (1991). Psychopharmacological evidence for a role of the ATP-sensitive potassium channel in the substantia nigra of the rat. Neuropharmacology 30: 359-365.

Madl JE, Burgesser K (1993). Adenosine triphosphate depletion reverses sodium-dependent, neuronal uptake of glutamate in rat hippocampal slices. J Neurosci 13: 4429-4444.

Maneuf YP, Duty S, Hille CJ, Crossman AR, Brotchie JM (1996). Modulation of GABA transmission by diazoxide and cromakalim in the globus pallidus: implications for the treatment of Parkinson's disease. Exp Neurol 139: 12-16.

Matute C, Alberdi E, Ibarretxe G (2002). Excitotoxicity in glial cells. Eur J Pharmacol 447: 239-246.

Meng CH, Ding JH, He HH, YANG Y, Hu G (2003). Relationship between neurotoxicity of 6-hydroxydopamine and glutamate transporter. Acta Pharmaceutica Sinica 38: 885-888.

Meshul CK, Emre N, Nakamura CM, Allen C, Donohue MK, Buckman JF (1999). Time-dependent changes in striatal glutamate synapses following a 6-hydroxydopamine lesion. Neuroscience 88: 1-16.

McGroarty A, Greenfield SA (1996). Blockade of dopamine storage, but not of dopamine synthesis, prevents activation of tolbutamide-sensitive $\mathrm{Kq}$ channel in the guinea-pig substantia nigra. Exp Brain Res 110: 360-366.

Mourre C, Widmann C, Lazdunski M (1990). Sulfonylureas binding sites associated with ATP-regulated Kq channels in the nervous system: autoradiographic analysis of their distribution and ontogenesis, and of their localization in mutant mice cerebellum. Brain Res 519: 29-43.

Murer MG, Dziewczapolski G, Menalled LB, Garcia MC, Agid Y, Gershanik O et al (1998). Chronic levodopa is not toxic for remaining dopamine neurons, but instead promotes their recovery, in rats with moderate nigrostriatal lesions. Ann Neurol 43: $561-575$.

Neusch C, Runde D, Moser A (2000). G proteins modulate D2 receptor-coupled $\mathrm{K}(\mathrm{ATP})$ channels in rat DArgic terminals. Neurochem Res 25: 1521-1526.

Nutt GJ, Hloford NH (1996). The response to levodopa in Parkinson's disease: imposing pharmacological law and order. Ann Neurol 39: 561-573.

Paxinos G, Watson C (1986). The Rat Brain in Stereotaxic Coordinates, 2nd edn. Academic Press: San Diego.

Perese DA, Ulman J, Viola J, Ewing SE, Bankiewicz KS (1989). A 6-hydroxydopamine-induced selective Parkinsonian rat model. Brain Res 494: 285-293.

Pines G, Kanner BI (1990). Counterflow of 1-glutamate in plasma membrane vesicles and reconstituted preparations from rat brain. Biochemistry 29: 11209-11214.
Roeper J, Hainsworth AH, Ashcroft FM (1990). Tolbutamide reverses membrane hyperpolarisation induced by activation of $D$ receptors 2 and GABA receptors in isolated substantia nigra neurons. Pflugers B Arch 416: 473-475.

Ronald D, Arjan B, Jos P (2002). Modeling Parkinson's disease in rats: an evaluation of 6-OHDA lesions of the nigrostriatal pathway. Exp Neurol 175: 303-317.

Tolwani RJ, Jakowec MW, Petzinger GM, Green S, Waggie K (1999). Experimental models of Parkinson's disease: insights from many models. Lab Anim Sci 49: 363-371.

Wang H (2003). Pharmacological characteristics of the novel antihypertensive drug, iptakalim hydrochloride, and its molecular mechanisms. Drug Dev Res 58: 65-68.

Wang H, Zhang YL, Tang XC, Feng HS, Hu G (2004). Targeting ischemic stroke with a novel opener of ATP-sensitive potassium channels in the brain. Mol Pharmacol 66: 1160-1168.

Wang S, Hu LF, Yang Y, Ding JH, Hu G (2005). Studies of ATP-sensitive potassium channels on 6-hydroxydopamine and haloperidol rat models of Parkinson's disease: implications for treating Parkinson's disease? Neuropharmacology 48: 984-992.

Yang Y, Liu X, Ding JJ, Sun J, Long Y, Wang F et al (2004). Effects of iptakalim on rotenone-induced cytotoxicity and dopamine release from PC12 cells. Neurosci Lett 366: 53-57.

Yang Y, Liu X, Long Y, Wang F, Ding JH, Liu SY et al (2005). Systematic administration of iptakalim, an ATP-sensitive potassium channel opener, prevents rotenone-induced motor and neurochemical alterations in rats. $J$ Neurosci Res 80: 442-449.

Yao HH, Ding JH, Zhou F, Wang F, Hu LF, Sun $\mathrm{T}$ et al (2005). Enhancement of glutamate uptake mediates the neuroprotection exerted by activating group II or III metabotropic glutamate receptors on astrocytes. J Neurochem 92: 948-961.

Vandenberg RJ (1998). Molecular pharmacology and physiology of glutamate transporters in the central nervous system. Clin Exp Pharmacol Physiol 25: 393-400.

Zhang Y, Ding JH, Hu G (2004). SIB-1893 reversed 6-OHDAinduced inhibitory effect on glutamate uptake of astrocytes. Chin J Clin Pharmacol Ther 9: 25-28.

Zhou M, Tanaka O, Suzuki M, Sekiguchi M, Takata K, Kawahara K et al (2002). Localization of pore-forming subunit of the ATPsensitive $\mathrm{K}(+)$-channel, Kir6.2, in rat brain neurons and glial cells. Brain Res Mol Brain Res 101: 23-32.

Zhu DX, Sullivan JP, Brioni JD (1999). ATP-sensitive potassium channels regulate in vivo DA release in rat brain. Jpn J Pharm 79: 59-64. 